\title{
Analysis of Outpatient Service Queue of Public Hospital in Jakarta
}

\author{
M. Dachyar*, Farizal, and M. Mansur Yafi \\ Industrial Engineering Department, Universitas Indonesia
}

\begin{abstract}
In recent years Public hospital services are becoming a burden in many large cities. Many patients are needed to be served, but it was constrained by the number of doctors. Even though the resources are limited, the efficiency of public hospital services is important. The patient's queues can be shortened by service quality enhancement of outpatient. This study has applied Business Process Reengineering (BPR) method to improve the efficiency of public hospital services. Igrafx software is used to visualize the improvement design. The result has suggested a solution to accelerate the outpatient health services of a public hospital in Jakarta.
\end{abstract}

\section{Introduction}

The quality of public hospital has to be improved as patients' demand grows [1]. The services quality improvement of the public hospital is different from the private one, especially in term of goals where private hospital are more profit-oriented [2]. The acceleration of outpatient services procedure, the public hospital can also increase patient satisfaction [3].

The public hospitals in a big city in Indonesia like Jakarta should get serious attention from the government in term of improvement. The patients could have been queuing since 5 am. This research is aimed to reduce the queues of outpatient service in public hospital in Jakarta. Based on the observation conducted between March and June 2017, the patient queues from 9 am to 10 am the queue on internal disease clinic around 110 patient, the queue on cardiac clinic around 104 patient and pulmonary clinic 60 patient.

\section{Literature Review}

Outpatient service process includes a registration process, medical examination, health treatment in the examination room, further examination, and received prescriptions [4]. Patient waiting time describes access to health services that become an indicator of health service quality. The waiting time potentially affects patient dissatisfaction with services, which closely related to the image of the hospital. The reason for giving the least attention to waiting time in the hospital is acceptable where service improvement is not a priority.

Queue occurs when objects to be served is confronted with delays of busyness service system. It happened when there is no balance between the serviced and services [5]. These

\footnotetext{
*Corresponding author: mdachyar@yahoo.com
} 
are the visualization of long queue where easily can find such as in teller transaction in the Bank, at the airport when passengers came in the check-in desk, in the supermarket queuing on paying goods, in a car wash where cars are queuing to be washed. In the service sector queuing is bored. Consequently, the costumers will leave. This is considered as a loss for the organization [6]. The best service among others is to provide quick service to the customers, so they don't wait too long. However, the impact of this quick service delivery will incur costs for organizations such as hospitals, as they have to add service facilities and technologies that are expensive.

Business process re-engineering (BPR) is a fundamental evaluation with a radical business process change for obtaining the improvement in main and contemporary performance indicator such as cost, quality, service, and speed [7]. In addition, according to Schonsleben business process re-engineering is the improvement of a business process by redesigning the process fundamentally [8]. Du Plessis also explained that BPR is a fundamental analysis and design of each process and radical business process including the business operation, the management system, the division of tasks, the structure, the principle, and the organizational behavior. The goal is to improve the performance in the interest of fulfilling business development demand also information technology development as the main stimulant of this process [9].

There were several companies that have implemented BPR, such as milk company [10], pharmaceutical company [11], shipping company [12].

\section{Research Methodology}

This research uses Business Process Re-engineering (BPR) method to map out outpatient service for reducing the outpatient service queue of a public hospital in Jakarta. BPR contains three (3) steps, which are the preparation for outpatient service improvement, the mapping and analysis of outpatient service process' initial condition, and the designing of outpatient service process improvement.

There are three main sub-processes in the outpatient service procedure, which are the registration, the polyclinic service, and the drug service. Based on the analysis using a Pareto diagram, three clinics were chosen as the research object. They are internal disease polyclinic, cardiac polyclinic, and pulmonary polyclinic.

\section{Results and Discussion}

\subsection{Prepare for Outpatient Service Process Reengineering}

The preparation phase is a stage for comprehending the initial condition of outpatient service process in public hospital and knowing the purpose of its business process.

\subsection{Mapping and Analysis of Outpatient Service Process Initial Condition}

Mapping the Initial Condition. In this phase, time study technique is used for achieving the standard time of each hospital service. After identifying the initial condition with process chart, the author creates the as-is process.

The patient registration process is to get the queue number for short time if they come with a complete document. Medical records were sought manually which takes about 30 minutes, then delivered to polyclinic. The next process is blood pressure measurement and body weight measurement is common to all the polyclinic. The patients will be examined by a doctor and prescribed for medicine at available pharmacies in the hospital. The process of 
taking the drug requires a little queue time because some drugs are not yet available and must be mixed manually first. The patient can go home and return as scheduled by the doctor. The patient queue occurs from 9-10 am at every clinic and pharmacy, after that the queue will be reduced. Based on queueing data simulation was developed, the average wait time for internal disease clinic is 6 hours 51 minutes, the average wait time for the cardiac clinic is 6 hours 46 minutes, the average wait time for the pulmonary clinic is 5 hours 45 minutes. The last step of analyzing phase is conducting an interview with experts to identify the root problems that need to be improved, such as:

1. The patient takes the queue number twice, for registration and the polyclinic

2. The prescription is still written manually.

3. The prescription copies are still written manually and inefficiently.

4. There is a long queue on polyclinic service.

5. Without verification on registration phase, the patient should automatically be registered in each polyclinic.

6. The pharmacists cannot access the patient's medication database directly.

7. The doctors and nurses are ready at the clinic but the medical records have not yet delivered to the clinic, because of the manual search of medical records.

\subsection{Design New Outpatient Service Process}

To reduce patient waiting time needs some improvement steps. Registration changed into electronic registration. The prescription process can be accelerated if the doctors input the data from every clinic. Increasing the number of doctors is one of the solutions to reduce queue time. Table 1 shows the suggested improvement that can be applied in the new design.

Table 1. Outpatient Service Improvement Suggestions

\begin{tabular}{|c|l|c|}
\hline No & \multicolumn{1}{|c|}{ Improvement } & Problem \\
\hline 1 & Online registration & 1 \\
\hline 2 & Printing the prescription & 2 \\
\hline 3 & Printing the prescription copies & 3 \\
\hline 4 & Increasing the number of doctors & 4 \\
\hline 5 & $\begin{array}{l}\text { Integrating the hospital information system with national } \\
\text { health insurance information systems }\end{array}$ & 5 \\
\hline 6 & Installation of prescription information system & 6 \\
\hline 7 & Installation of medical records information system & 7 \\
\hline
\end{tabular}

Table 2 shows stepwise improvement in three polyclinics.

Table 2. Four Steps of Service Improvement in Three Polyclinics

\begin{tabular}{|c|c|c|c|c|}
\hline \multirow{3}{*}{ Polyclinic } & \multicolumn{4}{|c|}{ Stage of improvement } \\
\hline & 1 & 2 & 3 & 4 \\
\hline & \multicolumn{4}{|c|}{ Improvements Combination } \\
\hline Internal disease clinic & 1,3 & 2,5 & 6 & 7 \\
\hline Cardiac clinic & 1,3 & $2,4,5$ & 4,6 & 7 \\
\hline Pulmonary clinic & 1,3 & 2,5 & 4,6 & 7 \\
\hline
\end{tabular}

The new service design is produced by combining some improvement suggestions. The service improvements consisted of several phases; the capability on implemented the technical capacity and financial capability of the organization. 
The simulation shows the greatest efficiency of each improvement stage as shown in Figure 1.

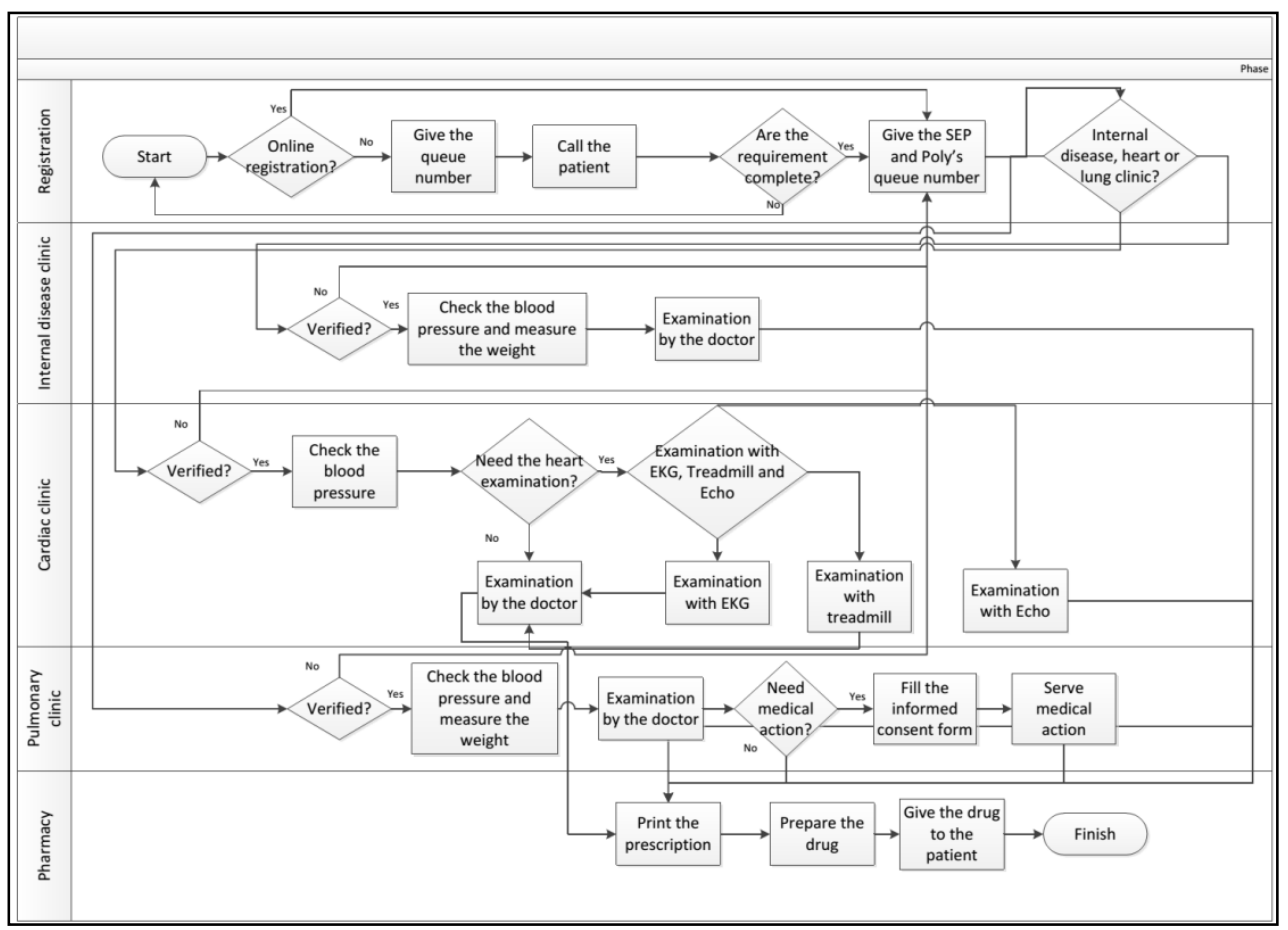

Fig. 1. Improved Outpatient Procedure

By implementing this improvement, internal disease clinic gained $21.7 \%$ efficiency time service process, cardiac clinic gained $22.5 \%$ and pulmonary clinic gained $21.8 \%$.

Table 3 shows the effectiveness of the service improvement, the initial condition and service improvement are simulated using the Igrafx software. The simulation result shows the outpatient service time reduction through the efficiency percentages Improvements are made in stages to determine the efficiency that occurs when the hospital can not yet make the comprehensive improvements over a short period of time due to hospital inadequacy in terms of technical, cost, and personnel. The service time in the hospital since the patient arrived to finish getting the drug takes more than 4 hours on average.

Patients who first come to the hospital when the total time of their service is not up to 4 hours, because waiting time in the polyclinic and in the pharmacy is still short, but this can happen if come to the hospital before 6 am, but this can be longer than 4 hours since later in the day.

Table 3. Process Improvement Simulation Result

\begin{tabular}{|c|c|c|c|c|}
\hline Polyclinic & $\begin{array}{l}\text { Current condition result } \\
\text { Avg Cycle (Hour) }\end{array}$ & Stage & $\begin{array}{l}\text { Improvements result } \\
\text { Avg Cycle (Hour) }\end{array}$ & Efficiency \\
\hline \multirow{4}{*}{ Internal disease clinic } & \multirow{4}{*}{7.34} & 1 & 6.83 & $6.90 \%$ \\
\hline & & 2 & 6.54 & $10.90 \%$ \\
\hline & & 3 & 6.22 & $15.30 \%$ \\
\hline & & 4 & 5.75 & $21.70 \%$ \\
\hline \multirow{2}{*}{ Cardiac clinic } & \multirow{2}{*}{7.33} & 1 & 6.63 & $9.50 \%$ \\
\hline & & 2 & 6.36 & $13.20 \%$ \\
\hline
\end{tabular}




\begin{tabular}{|l|l|l|l|l|}
\hline Polyclinic & $\begin{array}{l}\text { Current condition result } \\
\text { Avg Cycle (Hour) }\end{array}$ & Stage & $\begin{array}{c}\text { Improvements result } \\
\text { Avg Cycle (Hour) }\end{array}$ & Efficiency \\
\hline & & 3 & 6.14 & $16.20 \%$ \\
\cline { 3 - 5 } & & 4 & 5.68 & $22.50 \%$ \\
\hline \multirow{4}{*}{ Pulmonary clinic } & \multirow{2}{*}{6.09} & 1 & 5.57 & $8.50 \%$ \\
\cline { 3 - 5 } & & 2 & 5.28 & $13.30 \%$ \\
\cline { 3 - 5 } & & 3 & 5 & $21.80 \%$ \\
\cline { 3 - 5 } & & 4 & 4.76 & \\
\hline
\end{tabular}

\section{Conclusion}

This research resulted in the recommendation of a gradual improvement for three clinics on outpatient service of a public hospital in Jakarta. Internal disease polyclinic service time has been reduced from $6.9 \%-21.7 \%$. Cardiac polyclinic service time has been reduced from 9.5\%-22.5\%. Pulmonary polyclinic service time has been reduced from $8.5 \%-21.8 \%$. In order to reach less than 4 hours of service time, further research is needed.

This research funded by Universitas Indonesia - PITTA 2017

\section{References}

1. A. Shabbir, S. A. Malik, Int. J. of Q. \& Rel. Mgmt, 538-557 (2016).

2. J. S. Harrison \& S. M. Strategic Management of Healthcare Organizations. New York: Business Expert Press (2015).

3. Z. Lulu, M. Li, F. Ye, T. Ding, \& P. Kang, An Investigation Report on Large Public Hospital Reforms in China. Singapore: Springer (2016).

4. S. Chand, H. Moskowitz, J. B. Norris, S. Shade, \& D. Willis, H. C. Mgmt. Sci., 325-340 (2009).

5. L. J. Krajewski, L. P. Ritzman, \& M. K. Maholtra, Operations Management: Processes and Supply Chains. Essex: Pearson Education (2013).

6. J. A. Fitzsimmons \& M. J. Fitzsimmons, Service Management: Operations, Strategy, Information Technology. New York: McGraw-Hill (2010).

7. M. Hammer \& J. Champy, Reengineering The Corporation: A Manifesto for Business Revolution. Perfectbound (2001).

8. P. Schönsleben, Integral Logistics Management: Operations and Supply Chain Management Within and Across Companies. Boca Raton: CRC Press (2012).

9. S. Mohapatra, Business Process Reengineering. London: Springer (2013).

10. M. Dachyar \& E. Christy, J. of Phys. (2014).

11. M. Dachyar \& G. Novita, ARPN J. of Engr. and App. Sci. (2016).

12. M. Dachyar \& B. Praharani, Improvement of Procurement Business Process (Procure to Pay) in Indonesia Shipping Company. Knowledge, Service, Tourism \& Hospitality. CRC Press (2016). 